University of Nebraska - Lincoln

DigitalCommons@University of Nebraska - Lincoln

Robert G. Fuller Publications and Presentations Research Papers in Physics and Astronomy

December 1968

\title{
Ionic Transport in Potassium Chloride
}

Robert Fuller

rfuller@neb.rr.com

Charles L. Marquardt

Naval Research Laboratory, Washington, D. C.

Michael H. Reilly

Naval Research Laboratory, Washington, D. C.

John C. Wells Jr.

Naval Research Laboratory, Washington, D. C.

Follow this and additional works at: https://digitalcommons.unl.edu/physicsfuller

Part of the Physics Commons

Fuller, Robert; Marquardt, Charles L.; Reilly, Michael H.; and Wells, John C. Jr., "Ionic Transport in Potassium Chloride" (1968). Robert G. Fuller Publications and Presentations. 11.

https://digitalcommons.unl.edu/physicsfuller/11

This Article is brought to you for free and open access by the Research Papers in Physics and Astronomy at DigitalCommons@University of Nebraska - Lincoln. It has been accepted for inclusion in Robert G. Fuller Publications and Presentations by an authorized administrator of DigitalCommons@University of Nebraska - Lincoln. 


\title{
Ionic Transport in Potassium Chloride
}

\author{
Robert G. Fuller, Charles L. Marquardt, Michael H. Reilly,* and John C. Wells, Jr. $†$ \\ Naval Research Laboratory, Washington, D. C. 20390
}

\begin{abstract}
The electrical conductivity and chlorine ion diffusion in $\mathrm{KCl}$ and $\mathrm{KCl}: \mathrm{SrCl}_{2}$ single crystals have been analyzed by least-squares methods, using as a model a perfect crystal perturbed by five defects: isolated anion vacancies, isolated cation vacancies, divalent cation impurities, divalent cation-impurity-cationvacancy complexes, and vacancy pairs. The transport equations were derived from this five-defect model using a simple theory for noninteracting particles, except for the nearest-neighbor binding to form complexes and vacancy pairs, and using the same theory including long-range Coulomb interactions between the isolated defects. This latter theory yielded the better description of the experimental results. However, the analyses showed that significant nonrandom deviations exist between theory and experiment. These deviations exist in both the intrinsic and extrinsic regions of conductivity. The failure of existing concepts for these transport properties is discussed in terms of possible additional mechanisms, i.e., electrons, cationic Frenkel defects, or trivacancies, and in terms of more complete theoretical treatment.
\end{abstract}

\section{INTRODUCTION}

$I^{\mathrm{oN}}$ ON transport in alkali halides has been studied for many years by means of electrical conductivity and diffusion measurements. An excellent review of work in this field prior to 1957 has been written by Lidiard. ${ }^{1}$ Since that time a great deal of experimental work has been done using improved techniques, ${ }^{2}$ but, until recently, ${ }^{3-5}$ no improvement in the existing concepts of ionic transport had seemed necessary. A theory which has been most widely used to analyze transport data is a simple theory including association (STIA). This theory (described in Sec. I C below) takes into account cation vacancies, anion vacancies, and divalent cation impurities in the crystal lattice. It assumes that the defects are completely noninteracting unless two oppositely charged defects become nearest neighbors in the crystal lattice, in which case they are treated as a single defect having no net charge. The conductivity calculated from the STIA is a function of several temperature-independent parameters, representing defect formation and migration entropies and enthalpies. In the work of Beaumont and $\mathrm{Jacobs}^{6}$ on $\mathrm{KCl}$ the best values of these parameters were obtained by making a least-squares fit of the conductivity data to the STIA. A small systematic discrepancy between theory and experiment was not emphasized by these authors.

Nonetheless the inadequacy of the STIA was recognized by Lidiard as early as 1954 when he proposed the inclusion of long-range Coulomb interactions in the model. ${ }^{7}$ More recently, definite inconsistencies be-

* NRC-NRL Postdoctoral Research Associate.

$\dagger$ Present address: Radiation Physics Division, Naval Ordinance Laboratory, White Oak, Silver Spring, Md.

${ }^{1} \mathrm{~A}$. B. Lidiard, in Handbuch der Physik, edited by S. Flügge (Springer-Verlag, Berlin, 1957), Vol. XX, Part 2, pp. 246-349

2 P. Süptitz and J. Teltow, Phys. Status Solidi 23, 9 (1967).

${ }^{3}$ R. G. Fuller and M. H. Reilly, Phys. Rev. Letters 19, 113 (1967).

${ }^{4}$ R. G. Fuller, M. H. Reilly, C. L. Marquardt, and J. C. Wells, Jr., Phys. Rev. Letters 20, 662 (1968).

S A. R. Allnatt and P.' Pantelis, Solid State Commun. 6, 309 (1968).

${ }_{6}^{6}$ J. H. Beaumont and P. W. M. Jacobs, J. Chem. Phys. 45, 1496 (1966).

${ }^{7}$ A. B. Lidiard, Phys. Rev. 94, 29 (1954). tween experimental data and the STIA have been observed in non-Ohmic conductivity of several alkali halides ${ }^{8}$ as well as in the Ohmic conductivity of "pure" $\mathrm{KCl}^{4}$ and "pure" $\mathrm{NaCl} .{ }^{5}$ Thus, it has become increasingly clear that the STIA is not only incomplete from a theoretical point of view, but is also incapable of explaining the finer details of ionic conduction.

It must be noted that the deviation of the experimental data from the "best fit" of STIA as a function of temperature and divalent impurity concentration is relatively small. Thus, to test the theory, we must examine a large body of high-precision data which covers a wide range of temperatures and impurity concentrations. In the present work we have obtained such a body of conductivity data, using $\mathrm{KCl}$ and $\mathrm{KCl}: \mathrm{SrCl}_{2}$ crystals upon which measurements of self-diffusion of chlorine had previously been made. ${ }^{9}$ We have used these conductivity and diffusion data to test the STIA as well as an improved theory (described in Sec. II D below) which includes long-range Coulomb interactions (after Lidiard). ${ }^{7}$ It will be shown that the data agree somewhat better with the Lidiard theory than they do with the STIA, but there are non-negligible systematic deviations from both models.

\section{THEORY}

Electrical conduction in alkali halides is known to be primarily ionic. The conductivity as well as the selfdiffusion can be explained by the motion of cation and anion vacancies in the lattice. The diffusion coefficient for an isolated ion, in a $\mathrm{NaCl}$-type lattice is given by ${ }^{1}$

$$
D=4 f a^{2} n w,
$$

where $f$ is the correlation factor, $a$ is the anion-cation distance, $n$ is the mole fraction of vacancies, and $w$ is the probability per unit time that a vacancy will jump from one particular site to another. Using the Nernst-

\footnotetext{
${ }^{8}$ C. E. Skov and E. A. Perlstein, Phys. Rev. 137, 1483 (1965).

${ }^{9}$ R. G. Fuller, Phys. Rev. 142, 524 (1966).
} 
Einstein relation ${ }^{1}$ we may write the conductivity as

$$
\sigma=\frac{N e^{2} D}{f k T}=\frac{4 N e^{2} a^{2} n w}{k T},
$$

where $N$ is the number of ions (positive or negative) per unit volume, $e$ is the magnitude of electronic charge, and $k$ is the Boltzmann constant.

For calculating the actual ionic conductivity of $\mathrm{KCl}$, contributions of both cation and anion vacancies must be included. ${ }^{10}$ Thus, we obtain the sum of two terms each having the form of Eq. (2):

$$
\sigma T=\left(4 N e^{2} a^{2} / k\right)\left(n_{a} w_{a}+n_{c} w_{c}\right),
$$

where the subscripts $a$ and $c$ refer to anion and cation vacancies, respectively.

In the self-diffusion of chlorine in $\mathrm{KCl}$ it has been shown that there are contributions due to single anion vacancies and vacancy pairs. ${ }^{9}$ The latter consists of an anion vacancy bound to a nearest-neighbor cation vacancy. Hence, the diffusion equation for $\mathrm{Cl}^{36}$ tracer is the sum of two terms each having the form of Eq. (1), where the appropriate correlation factors take into account the nonrandomness of the tracer diffusion process :

$$
D_{\mathrm{Cl}}^{36}=D_{a}+D_{p}=4 f a^{2} n_{a} w_{a}+\frac{4}{3} f_{p} a^{2} n_{p} w_{p} .
$$

Here $f_{p}$ is the pair correlation factor, ${ }^{11} n_{p}$ is the mole fraction concentration of vacancy pairs, and $w_{p}$ is the probability per unit time that a vacancy pair will reorient by a jump of the anion-vacancy component.

The ionic conductivity and the chlorine ion diffusion in potassium chloride are related to the microscopic properties of the $\mathrm{KCl}$ single crystal through Eqs. (3) and (4). It is to be noted in both of these equations that the microscopic properties of $\mathrm{KCl}$ appear always as the product of the jump probability times the defect concentration.

\section{A. Jump Probability}

An expression for the jump probability for ions in ionic solids has been derived using several different approaches, ${ }^{12}$ e.g., absolute rate theory, many-body theory of equilibrium statistics, and the dynamical theory of diffusion. All these derivations have resulted in an expression of the following form:

$$
w=\nu \exp (-\Delta g / k T),
$$

where $\Delta g$ is the height of the free-energy barrier the ion must surmount to pass to the adjacent vacant site and $\nu$ is an effective vibration frequency of the ion in the initial site.

${ }^{10}$ A. R. Allnatt and P. W. M. Jacobs, Trans. Faraday Soc. 58, 116 (1962).

11 R. E. Howard, Phys. Rev. 144, 650 (1966).

12 H. R. Glyde, Rev. Mod. Phys. 39, 373 (1967).

\section{B. Defect Concentrations}

In Eqs. (1)-(4), we have introduced three mobile defects which give rise to ion transport in $\mathrm{KCl}$. In the case of alkali halides containing non-negligible amounts of alkaline-earth impurity two additional defects must be considered: substitutional divalent cation impurities and impurity-vacancy complexes. The latter consists of a divalent cation impurity and a cation vacancy occupying nearest-neighbor sites. Impurities are assumed to be immobile, but they play a role in determining the concentration of the mobile defects. The concentrations of the five defects can be determined by the use of equilibrium statistical mechanics. The results are well known for the case when the defects are treated as noninteracting particles. ${ }^{1}$ They are usually written as follows :

$$
\begin{gathered}
n_{a} n_{c}=\exp (-g / k T) \equiv n_{0}^{2}, \\
n_{k} /\left(n_{c}\left(n_{i}-n_{k}\right)\right)=12 \exp (\zeta / k T) \equiv K_{1}(T), \\
n_{p}=6 n_{c} n_{a} \exp \left(g_{b} / k T\right) \\
=6 \exp \left(-g_{p} / k T\right), \\
n_{c}=n_{a}+n_{i}-n_{k},
\end{gathered}
$$

where $g$ is the free energy for Schottky defect formation, $n_{i}$ is the mole fraction of divalent impurity concentration, $n_{k}$ is the mole fraction of divalent-impuritycation-vacancy complexes, $g_{b}$ is the binding energy of a vacancy pair, $g_{p}\left(=g-g_{b}\right)$ is the free energy of vacancy pair formation, and $\zeta$ is the free energy of binding for an impurity-vacancy complex.

The equations for the concentration of defects were generalized by Lidiard to include long-range Coulomb interactions in the Debye-Hückel approximation. ${ }^{7}$ The inclusion of Coulomb interactions does not change Eqs. (8) and (9). Hence, the generalized equations for defect concentrations can be written in a form similar to the above equations upon replacing Eq. (6) and (7) by the following:

$$
\begin{gathered}
n_{a} n_{c}=\exp (-(g-\gamma) / k T), \\
n_{k} / n_{c}\left(n_{i}-n_{k}\right)=12 e^{(\zeta-\gamma) / k T},
\end{gathered}
$$

where

$$
\begin{gathered}
\gamma=e^{2} \kappa /(1+2 \kappa a), \\
\kappa^{2}=32 \pi e^{2} n_{c} / \epsilon a^{3} k T,
\end{gathered}
$$

and $\epsilon$ is the static dielectric constant.

\section{Simple Theory Including Association}

The STIA uses the general equations for conductivity and diffusion, Eqs. (3) and (4), the expression for jump probability given by Eq. (5), and the expressions for the concentrations of mobile defects that result from combining Eqs. (6)-(9). The STIA permits exact calculation of conductivity and diffusion as functions of temperature and divalent cation-impurity concentra- 
tion in terms of eleven adjustable parameters as follows :

$$
\begin{aligned}
\sigma T= & \frac{4 N e^{2} a^{2} \nu}{k}\left[\frac{n_{a}}{n_{0}} \exp \frac{\left(s / 2+\Delta s_{a}\right)}{k} \exp \frac{\left[-\left(h / 2+\Delta h_{a}\right)\right]}{k T}\right. \\
& \left.+\frac{n_{0}}{n_{a}} \exp \frac{\left(s / 2+\Delta s_{c}\right)}{k} \exp \frac{\left[-\left(h / 2+\Delta h_{c}\right)\right]}{k T}\right],
\end{aligned}
$$

$D_{\mathrm{Cl}^{36}}=4 f a^{2}\left(\frac{n_{a}}{n_{0}}\right) \exp \left[s / 2 k+\Delta s_{a} / k-\left(h / 2+\Delta h_{a}\right) / k T\right]$

where

$$
+D_{p} \exp (-Q / k T)
$$

$$
\begin{aligned}
\left(n_{a} / n_{0}\right)^{3}+\left(n_{a} / n_{0}\right)^{2}\left[n_{i} / n_{0}+\right. & \left.K_{1}(T) n_{0}\right] \\
& -n_{a} / n_{0}-K_{1}(T) n_{0}=0
\end{aligned}
$$

and

$$
K_{1}(T)=12 \exp (-\eta / k+\chi / k T) .
$$

The eleven parameters are defined as follows:

$s$ is the entropy of Schottky defect formation,

$h$ is the enthalpy of Schottky defect formation,

$\Delta s_{a}$ is the entropy of anion migration,

$\Delta h_{a}$ is the enthalpy of anion migration,

$\Delta s_{c}$ is the entropy of cation migration,

$\Delta h_{c}$ is the enthalpy of cation migration,

$\eta$ is the entropy of divalent-impurity-cationvacancy association,

$\chi$ is the enthalpy of divalent-impurity-cationvacancy association,

$D_{p}$ is the preexponential factor for the vacancy pair contribution to the chlorine ion tracer diffusion,

$Q$ is the activation energy of the vacancy pair contributions to the chlorine ion tracer diffusion, and

$\nu$ is the effective vibration frequency of the ions in their equilibrium sites. ${ }^{13}$

To separate the details of the transport phenomena from known temperature effects, the temperature dependence of the preexponential constants in Eqs. (14) and (15) was taken into account in our analysis. This temperature dependence results from the changes of the lattice parameter and the lattice frequency with temperature. However, because of the experimental procedures, some of the temperature dependence can be neglected. ${ }^{14}$ The lattice frequency as a function of

${ }^{13}$ It is assumed that the effective vibration frequency around each of the two isolated vacancy types is the same. However, Takeno [in Lattice Dynamics, edited by R. F. Wallis (Pergamon Press, Inc., New York, 1965), p. 497] has found on a nearestneighbor force-constant model that there are localized gap modes associated with a vacancy at the heavier ion lattice site. In a first approximation, however, the parameters $s_{a}$ and $s_{c}$ include this effect in the fitting procedure (see Ref. 12).

14 The experimental value of the conductivity at temperature $T$ was calculated from its resistance measured at $T$ and its thickness over area ratio measured at room temperature. The thermal expansion correction to the $t / A$ ratio cancels the thermal expansion correction to the $\mathrm{Na}^{2}$ term on the right-hand side of Eq. (14) Similarly the experimental value of the tracer diffusion coefficient at temperature $T$ was calculated from the tracer penetration profile for a crystal sliced at room temperature. The thermal expansion temperature is estimated from the data for $\mathrm{LiF}$ and $\mathrm{MgO}$ to be $\nu=\nu_{0}(1+\beta T)$ with $\beta=2.5 \times 10^{-4}{ }^{\circ} \mathrm{C}^{-1}$ and $T$ in degrees centrigade. ${ }^{15}$

The preexponential constant for the vacancy pair contribution to anion diffusion is temperature-dependent because of the lattice parameter and the correlation factor. Howard ${ }^{11}$ has shown the dependence of the vacancy pair correlation factor on the ratio of the jump frequencies of the anion and cation vacancies in the pair. The ratio of jump frequencies can be estimated using the theoretical results of Tharmalingam and Lidiard $^{16}$ and assuming equal activation entropies. When this is done the following equation is obtained for the ratio of the jump frequency of the cation vacancy in a vacancy pair in $\mathrm{KCl}$ to the jump frequency of the anion vacancy in a vacancy pair:

$$
w_{+} / w_{-}=e^{-\left(0.15 \mathrm{eV} / k T^{\prime}\right)} \text {. }
$$

The value of this ratio varies from 0.11 at $523^{\circ} \mathrm{C}$ to 0.18 at $723^{\circ} \mathrm{C}$. According to the results of Howard this would give the pair correlation factor a value of 0.30 at $523^{\circ} \mathrm{C}$ and of 0.38 at $723^{\circ} \mathrm{C}$. (It is of interest to note that the equivalent vacancy pair correlation factors for the vacancy pair contribution to cation diffusion in $\mathrm{KCl}$ would be 0.76 and 0.78 . This effect was previously discussed in regard to cation diffusion in $\mathrm{NaCl} .{ }^{17}$ ) Although the vacancy pair correlation may increase by almost $25 \%$ over the temperature range for which the diffusion data were taken, the temperature dependence of $D_{p}$ has a negligible effect on the values of the anion and cation vacancy and the impurityvacancy complex parameters. Hence, $D_{p}$ was treated as a constant in our analyses.

\section{Lidiard Theory}

The Lidiard theory generalizes the simple theory to include Coulomb interactions in the Debye-Huickel approximation and can be used with only slight modifications in Eqs. (14)-(17). The Coulomb interactions, in this approximation, may be included by substituting $h / 2-\gamma$ for $h / 2$ in Eqs. (14) and (15), by using Eq. (10) for the values of $n_{0}$, and by replacing $\chi$ in Eq. (17) by $\chi-\gamma$. In his original work ${ }^{6}$ Lidiard also incorporated a mobility drag factor $d$ which expresses the hindrance to ion motion by the Debye-Hückel charge cloud. This factor is given by

$$
d=1-e^{2} \kappa / 7.243 \epsilon k T\left(1+4.828 \kappa a+4 \kappa^{2} a^{2}\right) .
$$

The mobility drag can be incorporated into the transport equations by multiplying the right-hand side of Eq. (14) and the first term on the right-hand side of

correction to the slice thickness cancels the thermal expansion correction to the $a^{2}$ term on the right-hand side of Eq. (15).

${ }_{15}$ J. R. Jasperse, A. Kahan, J. N. Plendl, and S. S. Mitra, Phys. Rev. 146, 526 (1966).

${ }_{16} \mathrm{~K}$. Tharmalignam and A. B. Lidiard, Phil. Mag. 6, 1157 (1961).

17 A. B. Lidiard, J. Phys. Chem. Solids 6, 298 (1958). 
Eq. (15) by $d$. The inclusion of the Coulomb interactions according to Lidiard's equations permits the exact calculation of conductivity and diffusion as functions of temperature and divalent impurity concentration in terms of the same eleven adjustable parameters as the STIA (although the functional forms differ).

In addition to the temperature corrections discussed in Sec. II C, the temperature dependence of the static dielectric constant in Eq. (13) was included in our analyses using the Lidiard theory. The dependence of the dielectric constant on temperature was obtained from von Hippel's high-temperature data for $\mathrm{KCl}^{18}$ and was expressed as

$$
\frac{\epsilon-1}{\epsilon+2}=0.512 \exp \left(2.18 \times 10^{-4} T\right),
$$

where $T$ is the temperature in degrees centrigrade.

\section{EXPERIMENTAL PROCEDURE}

Single crystals of "pure" Harshaw $\mathrm{KCl}$ and $\mathrm{KCl}$ containing various concentrations of $\mathrm{SrCl}_{2}$ were obtained from the Materials Research Laboratory, University of Illinois. These were the same crystals for which the chlorine ion diffusion data had been reported. ${ }^{9}$ Samples about $1 \mathrm{~mm}$ thick and $5 \mathrm{~mm}$ square were cleaved from the crystals used in the diffusion measurements. For the conductivity measurements a sample was placed between the Pt electrodes of the conductivity cell. The electrodes were held against the sample with light pressure. The conductivity cell was evacuated, filled with dry argon, and placed in an electric furnace regulated by an electronic temperature controller with a platinum sensing resistor. The sample was annealed by thermal cycling from 680 to $720^{\circ} \mathrm{C}$ for several days to ensure good electrical contact between the electrodes and the sample. During the annealing procedure the resistance of the sample (at a given temperature) decreased monotonically to a definite value, $R$, such that $\Delta R / R$ was less than $1 \%$ for successive periods of thermal cycling process. When this condition was attained, good electrode-sample contact was assumed and the measurement of the electrical resistance of the sample as a function of temperature was begun. The Ohmicity of contacts made in this way was tested, and no detectable deviation from current-voltage linearity was found for fields up to $30 \mathrm{~V} / \mathrm{cm}$.

Resistance of the sample was measured over the temperature range $480^{\circ} \mathrm{C}<T<750^{\circ} \mathrm{C}$ with intervals of about $3^{\circ}$ between data points. This data density (number of data points per temperature interval) is at least four or five times greater than that reported in most of the previous conductivity work..$^{19}$ The taking

${ }^{18}$ A. R. von Hippel, U. S. Office of Naval Research, Technical Report No. 119, Massachusetts Institute of Technology, 1957, p. 30 (unpublished).

${ }^{19} \mathrm{See}$, for example, papers listed in Ref. 2. of high-density data was facilitated by our furnace design which combined large thermal mass with cooling by air flow through stainless steel tubing embedded in the insulating material around the heating element. This design permitted rapid change of temperature by small increments, using the cooling air, and still provided the temperature stability required for these measurements.

The resistance of the sample was measured at $1 \mathrm{kHz}$ using a General Radio Co. capacitance bridge, Type 716-C, and a General Radio tuned amplifier-null detector, Type 1231-A. The precision of the bridge was checked using standard resistors and was found to be about $\pm 0.3 \%$. The temperature of the sample was taken to be the average of the two temperatures measured by the Pt-PtRh thermocouples on opposite sides of the sample. The accuracy of the temperature measurements was estimated to be $\pm 0.5^{\circ} \mathrm{C}$ based on a calibration of these thermocouples after the completion of all our conductivity measurements.

After each resistance measurement was completed the thickness and area of the sample were measured. The thickness of the sample was measured using a micrometer caliper. The area of the sample was determined from a $20 \times$ photograph of the sample surfaces. The accuracy of the thickness and area measurement was estimated to be $1 \%$. The probable error of the conductivity calculated from the resistance, thickness, and area was estimated to be $2 \%$.

During data runs, when a sample was left overnight at moderate temperatures, it would show a small but detectable increase of resistance $(<1 \%)$. This change was irreversible; it could not be removed upon annealing at high temperatures. In fact, high-temperature resistances measured after the change were increased by a constant factor. This effect probably resulted from the change in the contact area between the $\mathrm{KCl}$ crystal and the Pt electrode. Data taken after the change was multiplied by an approrpiate correction factor so as to bring all values of resistance at high temperatures into agreement.

In a similar fashion the samples containing $\mathrm{Sr}$ were left overnight at low temperatures to determine the solubility limits for $\mathrm{Sr}$ in $\mathrm{KCl}$. Only the data from the regions of total $\mathrm{Sr}$ solubility were used in the conductivity analyses.

The concentration of $\mathrm{Sr}$ in the sample boules was determined by atomic abosrption. The results of the analyses are shown in Table I. Because of the effect of micro segregation of impurities these concentrations may be somewhat different from the actual concentrations in the conductivity samples. In the case of the heavily doped crystal it was possible to analyze the $\mathrm{Sr}$ content of the actual conductivity sample after the completion of the conductivity measurements. It was found that the sample contained $375 \pm 10 \mathrm{ppm} \mathrm{Sr}$.

The experimental data for $\mathrm{Cl}^{36}$ diffusion in $\mathrm{KCl}$ have 
TABLE I. Strontium content of the $\mathrm{KCl}$ crystals.

\begin{tabular}{|c|c|c|}
\hline \multirow{2}{*}{$\begin{array}{c}\text { Crystal } \\
\text { identification }\end{array}$} & \multicolumn{2}{|c|}{$\mathrm{ppm}$ mole fraction in crystals } \\
\hline & Boule & $\begin{array}{c}\text { Diffusion }^{b} \\
\text { samples }\end{array}$ \\
\hline Harshaw & 1 & $0.8 \pm 0.4$ \\
\hline D & $29 \pm 2$ & $21.0 \pm 0.4$ \\
\hline $\mathrm{C}$ & & $43.9 \pm 0.4$ \\
\hline A & $375 \pm 10^{\circ}$ & $237 \pm 7$ \\
\hline B & & $469 \pm 4$ \\
\hline
\end{tabular}

a Measured by R. W. Black at Naval Research Laboratory.

b Measured at Ames Laboratory, Ames, Iowa.

c Actual conductivity sample.

been reported. ${ }^{9}$ The following high-temperature diffusion data, which were free from anomalous effects (see Ref. 9), were used in this analysis: Harshaw $\mathrm{KCl}$, 20 data points for $T>600^{\circ} \mathrm{C} ; \mathrm{KCl}: \mathrm{SrCl}_{2}(21 \mathrm{ppm}), 17$ data points for $T>540^{\circ} \mathrm{C} ; \mathrm{KCl}: \mathrm{SrCl}_{2}(43.9 \mathrm{ppm}), 14$ data points for $T>560^{\circ} \mathrm{C} ; \mathrm{KCl}: \mathrm{SrCl}_{2}(237 \mathrm{ppm}), 8$ data points for $T>640^{\circ} \mathrm{C}$; and $\mathrm{KCl}: \mathrm{SrCl}_{2}(469 \mathrm{ppm})$, 8 data points for $T>640^{\circ} \mathrm{C}$. The probable errors of the measurements were estimated to vary from 8 to $14 \%$ for the diffusion coefficients and to be $\pm 2{ }^{\circ} \mathrm{C}$ for the temperatures.

\section{ANALYSIS OF DATA}

One of the most critical steps in conductivity and diffusion experiments is devising an analytical method whereby the desired information can be extracted from the experimental data. In earlier work graphical methods were used. But as was pointed out by Jain and Dahake ${ }^{20}$ and Beaumont and Jacobs ${ }^{6}$ such methods always involve making simplifying approximations, several of which are not easily justified. In more recent work $^{3-6,9}$ the method of least squares involving computer techniques has been used. In this method a theoretical model is used to determine the functional dependence of the conductivity and diffusion on certain parameters. The values of these parameters are then varied to determine a best fit in the least-squares sense. If the fit so obtained is good the standard deviation is small, the individual deviations are randomly distributed about zero, and the model is assumed to be adequate. If on the other hand the fit is not good, i.e., the standard deviation is large and/or the individual deviations are not random, the assumed model is not adequate over the whole range and the values of the parameters determined by the least-squares analysis are not necessarily of great physical significance.

The schemes for applying a least-squares analysis to a body of conductivity and diffusion data may be divided into three general classes:

1. Individual fitting methods in which the conductivity or diffusion data from each sample are analyzed separately.

${ }^{20}$ S. C. Jain and S. L. Dahake, Indian J. Pure Appl. Phys. 2, 71 (1964).
2. Group fitting methods in which all the conductivity are analyzed simultaneously and all the diffusion data are analyzed simultaneously.

3. Total data fitting methods in which all the conductivity and diffusion data are analyzed simultaneously.

Although the methods of class 3 seem to be the most objective, they encounter the practical problem that conductivity and diffusion data are of significantly different character, i.e., they differ in accuracy, in number of data points available, and in sensitivity to change in the values of the parameters of the model.

The analysis performed by Beaumont and Jacobs ${ }^{6}$ was done only for conductivity data and was done using the methods from class 1 . In the present work, a leastsquares analysis of all the diffusion data (67 points) and all the conductivity data (220 points) was performed using a combination of class-1 and class-2 methods. After having tried several differentl fitting schemes we adopted the procedure cycle out ined in Fig. 1, as it seemed least likely to introduce errors because of the fitting process.

Two difficulties in this analysis are the lack of an accurate determination of the total divalent cationimpurity concentration in the lightly doped and "pure" samples and the fact that both conductivity and diffusion are insensitive to changes in the values of certain parameters. As a consequence, in the procedure cycle (Fig. 1) the anion migration parameters are not permitted to vary in the conductivity analyses and the Schottky defect and complex formation parameters are not allowed to vary in the diffusion analyses. The parameters that were varied and the number of data points used in each analysis are shown in Fig. 1. The impurity concentrations of some lightly doped and "pure" samples were allowed to vary to insure that inaccurate impurity concentrations were not forcing the parameters into erroneous values.

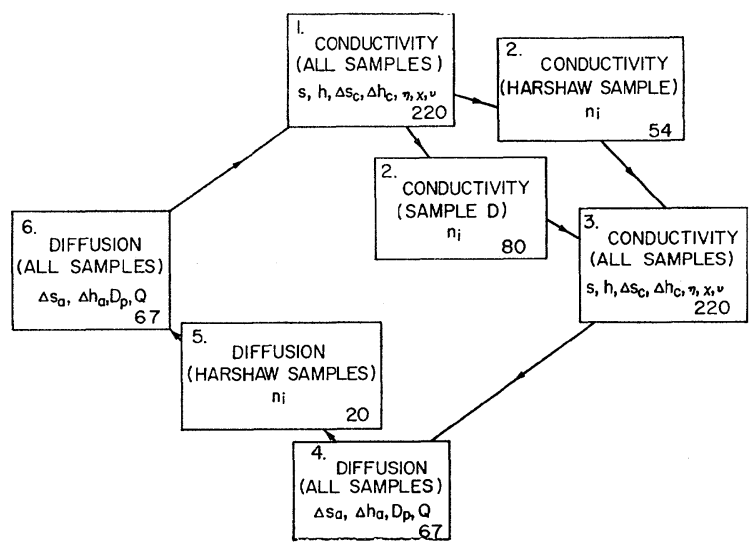

FIG. 1. Procedure cycle for the computer analysis. Each box represents a different analysis. The parameters listed in each box are the ones varied during that analysis. The number of data points in each analysis are given in the lower corner of each box. 
In each analysis the best parameter values were obtained by minimizing the function $W$, given by

$$
W=\sum_{k=1}^{N} \frac{\left(F_{\text {theor }}-F_{\text {expt }}\right)_{k}{ }^{2}}{N(\text { probable error })_{k}{ }^{2}},
$$

where $N$ is the number of data points and $F$ is the transport property (either conductivity or diffusion) computed and measured at the temperature $T_{k}$. The $W$ function was minimized by an iteration procedure where the value of the $i$ th parameter $(1 \leqslant i \leqslant 11)$ after the $j$ th iteration was given by

$$
\begin{array}{r}
P_{i}(j)=P_{i}(j-1)-\left[\left(\partial W / \partial P_{i}\right) /\left|\partial^{2} W / \partial P_{i}^{2}\right|\right] \\
P_{i}=P_{i}(j-1) .
\end{array}
$$

One complete procedure cycle consisted of the following steps (see Fig. 1):

1. Conductivity (all samples)-Parameters $s, h$, $\Delta h_{c}, \Delta s_{c}, \chi, \eta$, and $\nu$ were varied for a best fit of all the conductivity data. During this step $\Delta s_{a}, \Delta h_{a}, D_{p}, Q$, and all the impurity concentrations were held at fixed values [in fact, the conductivity analysis is independent of the values of $D_{p}$ and $Q$ as, the reader may easily verify from Eqs. (14), (16), and (17)].

2. Conductivity (sample Harshaw or $D$ )-In this step the conductivity data for an individual sample were fit using only one variable, the impurity concentration $n_{i}$. The eleven parameters were held fixed at the values they had at the end of step 1.

3 . Repeat step 1 with the corrected impurity concentrations obtained in step 2.

4. Diffusion (all samples)-Parameters $\Delta s_{a}, \Delta h_{a}, D_{p}$, and $Q$ were varied for a best fit to all the diffusion data. During this step $s, h, \Delta s_{c}, \Delta h_{c}, \chi, \eta$, and $\nu$ were held fixed at the values they had at the end of step 3 . The anion diffusion analysis is independent of the values of $\Delta s_{c}$ $\Delta h_{c}$ from Eqs. (15), (16), and (17). All the impurity concentrations were held fixed.
5. Diffusion (Harshaw samples)-In this step the diffusion data for the Harshaw samples were fit with only one variable, the impurity concentration. All the other parameters were held fixed at the values they had at the end of step 4.

6. Repeat step 4 using the corrected impurity concentrations for the Harshaw samples.

The cycle having been completed, a new set of eleven parameters and new impurity concentrations for the conductivity samples Harshaw and $D$ and for the Harshaw diffusion samples have been obtained. These new values gave a better fit to the experimental data than did the previous values and were used as starting values for the next cycle. The procedure was continued in cyclic fashion until the $W$ function for the conductivity (all samples) analysis was changing by less than $1 \%$ per cycle. This criterion was applied because the conductivity data were more numerous and more precise than the diffusion data and hence were more sensitive to small changes in the values of the parameters. A fit to the conductivity data implied a fit to all the data since the diffusion fit did not change appreciably after the first few cycles.

The above procedure required that a set of parameters be initially assumed. Several analyses were performed using different sets of initial parameters and using procedures similar to that outlined above and in Fig. 1. These complete anslyses, each requiring about $6 \mathrm{~h}$ of CDC 3800 computer time, were made for both the Lidiard theory (Sec. II D) and the STIA (Sec. II C). The median values and maximum variation in values of the eleven parameters as determined from these analyses are shown in Table II along with the results from other experiments.

In an attempt to pinpoint any deficiencies in the fivedefect model and the accompanying theories, the parameter values shown in Table II were used to calculate the

\begin{tabular}{|c|c|c|c|}
\hline Quantity & Lidiard theory & STIA & Others \\
\hline Schottky defect formation enthalpy, $h(\mathrm{eV})$ & $2.49_{-0.02}+0.03$ & $2.59_{-0.02}+0.18$ & $2.26^{\mathrm{a}}, 2.31^{\mathrm{b}}$ \\
\hline Entropy, $s / k$ & $7.64-0.02+0.24$ & $9.29-0.02+2.09$ & $5.37 \mathrm{a}, 7.56^{\mathrm{b}}$ \\
\hline Anion vacancy migration enthalpy, $\Delta h_{a}(\mathrm{eV})$ & $0.89_{-0.05}^{+0.01}$ & $0.82_{-0.17}+0.03$ & $0.95^{\mathrm{b}}, 1.04^{\mathrm{a}}$ \\
\hline Entropy, $\Delta s_{a} / k$ & $3.95_{-0.57}^{-0.05}+0.71$ & $2.94_{-1.98}+0.34$ & $6.28^{\mathrm{a}}, 1.04$ \\
\hline Cation vacancy migration enthalpy, $\Delta h_{c}(\mathrm{eV})$ & $0.76_{-0.01}+0.02$ & $0.79_{-0.01}+0.01$ & $0.71^{\mathrm{a}}, 0.84$ \\
\hline Entropy, $\Delta s_{c} / k$ & $2.56_{-0.04}+0.11$ & $2.86_{-0.18}+0.01$ & $1.89^{\mathrm{a}}$ \\
\hline $\begin{array}{l}\text { Divalent cation-impurity-cation-vacancy binding } \\
\text { enthalpy, } \chi(\mathrm{eV})\end{array}$ & $0.57_{-0.02}^{-0.04}+0.03$ & $0.36_{-0.03}+0.06$ & $0.42^{\mathrm{a}}$ \\
\hline $\begin{array}{l}\text { Entropy, } \eta / k \\
\text { Vacancy pair contribution to anion diffusion } \\
\text { activation energy, } O(\mathrm{eV})\end{array}$ & $\begin{array}{l}1.88_{-0.79}+1.60 \\
2.62_{-0.01}^{+0.01}\end{array}$ & $\begin{array}{l}0.52_{-0.16}+1.25 \\
2.59_{-0.11}+0.07\end{array}$ & $\begin{array}{l}0.0^{\mathrm{d}} \\
2.65^{\mathrm{b}}\end{array}$ \\
\hline $\begin{array}{l}\text { Diffusion constant, } D_{p}\left(\mathrm{~cm}^{2} / \mathrm{sec}\right) \\
\text { Jump attempt frequency, } \nu\left(\mathrm{sec}^{-1}\right) \\
\text { Average rms fractional deviation for analysis of } \\
\text { conductivity (all samples) }\end{array}$ & $\begin{array}{l}5150_{-3460}+150 \\
6.95_{-0.35}^{+0.35} \times 10^{12} \\
2.4 \%^{12}\end{array}$ & $\begin{array}{l}5430-4140^{+4150} \\
6.81_{-0.06}^{+0.36} \times 10^{12} \\
3.0 \%^{-12}\end{array}$ & $\begin{array}{l}8560^{\mathrm{b}} \\
4.25 \times 10^{12 \mathrm{c}}\end{array}$ \\
\hline
\end{tabular}
difference between the theoretical and experimental

TABLE II. Results of the analysis of the conductivity and diffusion data.

a Reference 6.

o R. W. Dreyfus and A. S. Nowick, J. Appl. Phys. 33, 473 (1962).

d Assumed value, see Ref. 6 . 


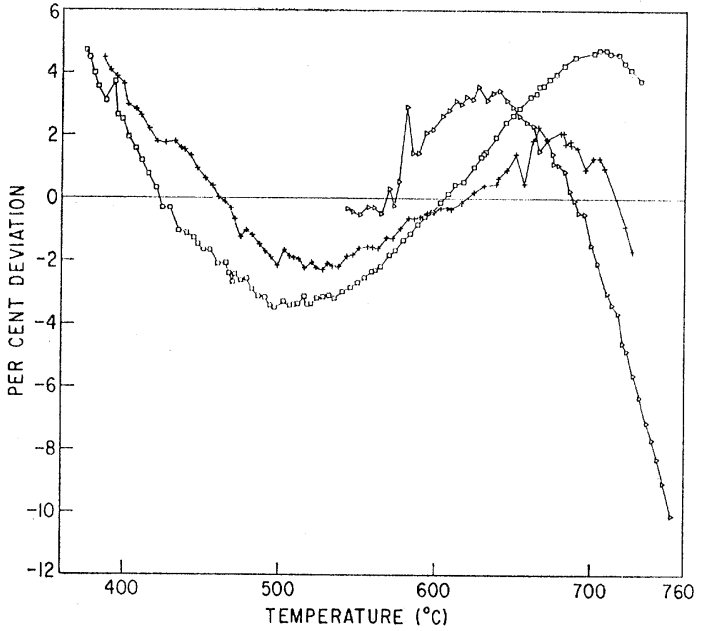

FIG. 2. Percent deviation as a function of temperature using STIA. $\Delta$, the percent deviation between the calculated and the measured values of conductivity for the Harshaw sample $(0.6$ ppm computed divalent cation impurity),+ , the percent deviation between the calculated and measured values of conductivity for the sample $D$ (16 ppm computed divalent cation impurity); $\square$, the percent deviation between the calculated and measured values of conductivity for the sample containing $375 \mathrm{ppm} \mathrm{Sr}$ by atomic absorption analysis.

values of the conductivity. The percent deviation, defined by the following equation:

Percent deviation

$$
=100 \times\left[(\sigma T)_{\mathrm{eale}}-(\sigma T)_{\operatorname{expt}}\right] /(\sigma T)_{\operatorname{expt}},
$$

is shown as a function of temperature for the STIA in Fig. 2. Similar curves obtained using the Lidiard theory

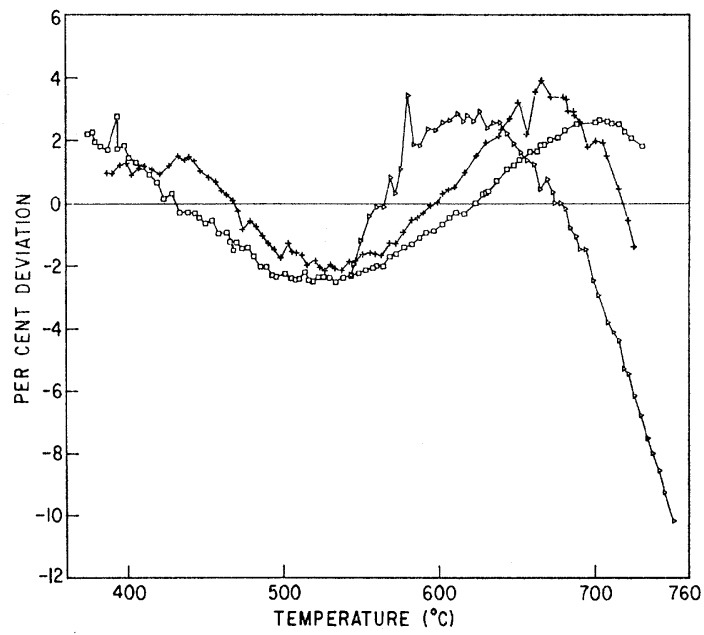

FIG. 3. Percent deviation as a function of temperature using the Lidiard theory. $\Delta$, the percent deviation between the calculated and measured values of conductivity for the Harshaw sample $(0.5$ ppm computed divalent impurity concentration); + , the percent deviation between the calculated and measured values of conductivity for the sameple $D(15 \mathrm{ppm}$ computed divalent impurity concentration); $\square$, the percent deviation between the calculated and measured values of conductivity for the sample containing $375 \mathrm{ppm}$ Sr by atomic absorption analysis. are shown in Fig. 3. Figure 4 compares the percent deviations obtained for one of the samples of Figs. 2 and 3 , the crystal containing $375 \mathrm{ppm} \mathrm{Sr}$, using the STIA and the Lidiard theory. The Lidiard theory evidently provides a slightly better description of the experimental data then the STIA. The average rms deviation obtained in the least-squares analyses of the conductivity (all samples) was $2.4 \%$ using the Lidiard theory and was $3.0 \%$ using the STIA. However, neither the Lidiard theory nor the STIA enable the five-defect model to give a precise quantitative description of the conductivity data. Neither set of equations fits the curvature of the $\log (\sigma T)$ versus $10^{3} / T$ plot in the intrinsic region of the conductivity as both fall some $10 \%$ below the experimental data near $750^{\circ} \mathrm{C}$. (See Figs. 2 and 3.) Similarly, neither set of equations provides

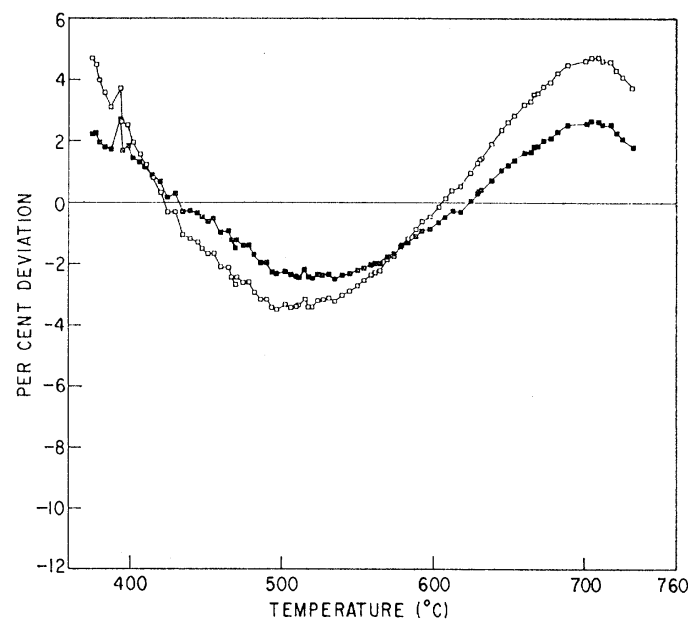

Fig. 4. Percent deviation as a function of temperature for the sample containing $375 \mathrm{ppm}$ Sr using the Lidiard theory and STIA. $\square$, the percent deviation between the calculated and the measured values of conductivity using the Lidiard theory; $\square$, the percent deviation between the calculated and measured values of conductivity using the STIA.

enough curvature to explain the extrinsic behavior of the $\mathrm{KCl}: \mathrm{SrCl}_{2}$ sample containing $375 \mathrm{ppm} \mathrm{Sr}$. The calculated values of the conductivity in the extrinsic region are low near $550^{\circ} \mathrm{C}$ and are high near 700 and $400^{\circ} \mathrm{C}$, resulting in a $\log (\sigma T)$ versus $10^{3} / T$ curve that is too nearly linear to describe the experimental results.

Nonrandom deviations evidently exist between the conductivity results and those of the STIA and the Lidiard theory for the five-defect model of alkali halide single crystals. In the view of this an extended discussion of the meaning of the values obtained for the various parameters, as shown in Table II, in this work and in the work of others would seem to be artificial. Let it suffice to note that there is general agreement between the various results, that the value we obtained for the formation enthalpy of the Schottky defects is rather large, about $2.5 \mathrm{eV}$, and that our jump 
frequency corresponds to an Einstein temperature of approximately $330^{\circ} \mathrm{K}$.

\section{DISCUSSION}

Ionic transport in $\mathrm{KCl}$ and $\mathrm{KCl}: \mathrm{SrCl}_{2}$ single crystals is presently understood in terms of five point defects, i.e., isolated anion vacancies, isolated cation vacancies, isolated divalent impurity cations, divalent impuritycation-cation-vacancy complexes, and vacancy pairs. The five-defect model is capable of describing the qualitative features of the diffusion and conductivity data, e.g., the change of the $D_{\mathrm{Cl}}$ at constant temperature as a function of $\mathrm{Sr}$ concentration (Fig. 5) and the curvature of the $\log (\sigma T)$ versus $10^{3} / T$ plots (Fig. 6).

The diffusion of $\mathrm{Cl}^{36}$ in $\mathrm{KCl}$ and $\mathrm{KCl}: \mathrm{SrCl}_{2}$ single crystals is described by the sum of a vacancy pair contribution and a single anion vacancy contribution (Eq. 4). The vacancy pair contribution to the diffusion is independent of the impurity concentration. A plot of the diffusion coefficient at constant temperature as a function of the impurity concentration shows the relative importance of the two contributions to the diffusion. Such a plot for the diffusion of $\mathrm{Cl}^{36}$ in $\mathrm{KCl}: \mathrm{SrCl}_{2}$ crystals at $670^{\circ} \mathrm{C}$ is shown in Fig. 5. The contributions

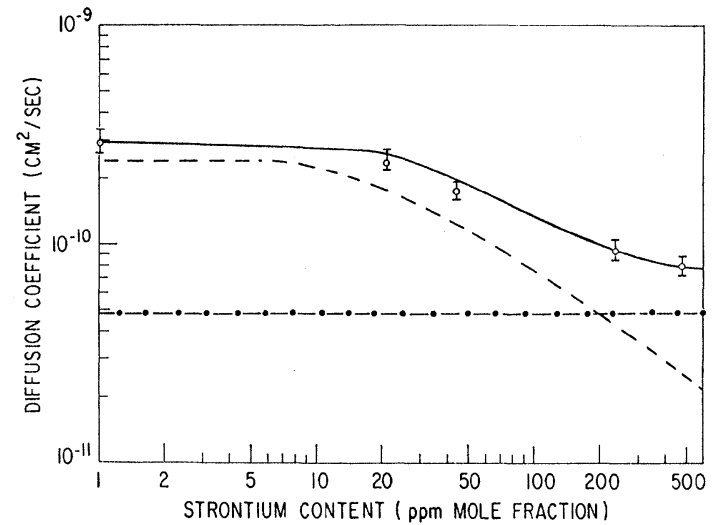

Frg. 5. The diffusion coefficient for chlorine ion diffusion in $\mathrm{KCl}$ and $\mathrm{KCl}: \mathrm{SrCl}_{2}$ single crystals as a function of strontium concentration. - - - - , the single anion vacancy contribution to the chlorine diffusion, see Eq. (4); -...-.-, the vacancy pair contribution to the chlorine ion diffusion, see Eq. (4).

from vacancy pairs and single anion vacancies as calculated from the parameter values given in the first column of Table II are also shown in Fig. 5.

The conductivity data for "pure" $\mathrm{KCl}$ and $\mathrm{KCl}: \mathrm{SrCl}_{2}$ crystals are shown in Fig. 6 in the usual $\log (\sigma T)$ versus

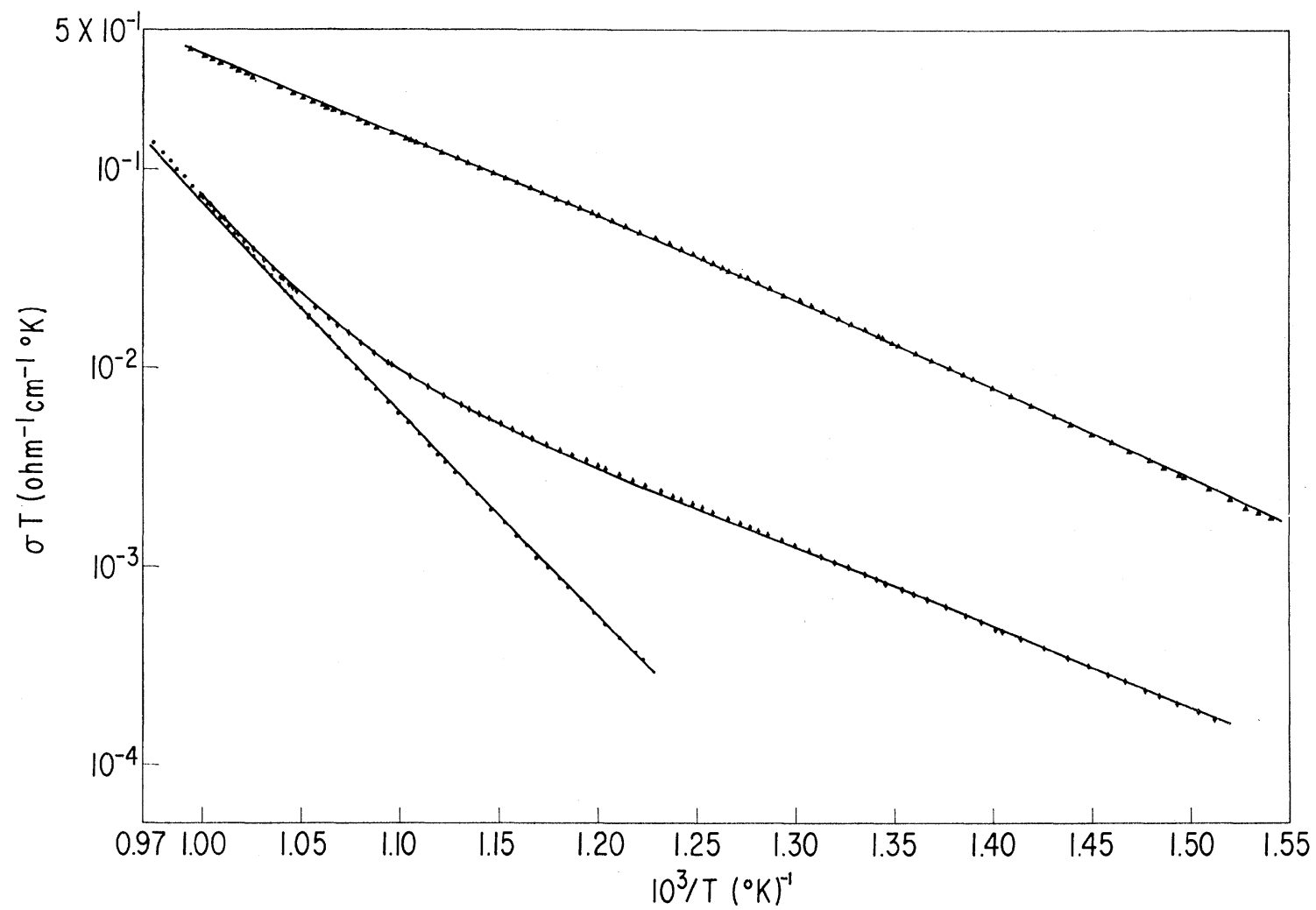

FIG. 6. The conductivity-absolute temperature product as a function of reciprocal temperature. The circles represent the data for the Harshaw sample. The diamonds represent the data for sample $D$ (approximately $15 \mathrm{ppm} \mathrm{Sr}$ ). The triangles represent the data for the sample containing $375 \mathrm{ppm}$ Sr. The solid lines are the results of the computer analysis using the parameters given in Table II, çolụmn 1, 
$10^{3} / T$ graph. The qualitative features of these plots are in agreement with data previously reported for this system. The positive curvature of the "pure" $\mathrm{KCl}$ plot was first noted and explained by Allnatt and Jacobs ${ }^{10}$ as a direct consequence of the sum of two exponential terms in Eq. (3). The negative curvature of the $\mathrm{KCl}$ : $\mathrm{SrCl}_{2}$ plots results from the well-known association effect, ${ }^{21,22}$ i.e., the formation of divalent cation-impuritycation-vacancy complexes. But the quantitative agreement between the data and the theories is not precise and the deviation between theory and experiment is not random (see Figs. 2, 3, and 4).

The fact that next-nearest-neighbor (NNN) complexes may also be present and make a contribution to the right-hand side of Eq. (17) in the form of $6 \exp \left(\zeta_{2} / k T\right)$ was considered. Our analysis using the Lidiard theory was modified to include such a term and two complete analyses (Fig. 1) were made. The average results of the two runs gave the following values for NNN complex binding enthalpy and entropy: $\chi_{2}=0.56$ $\pm 0.01 \mathrm{eV}$ and $\eta_{2} / k=2.3 \pm 0.6$. However, the character of the nonrandom deviations between theory and experiment was not changed by the inclusion of NNN complexes.

Several possible explanations for these nonrandom deviations between the conductivity results and those of the STIA and the Lidiard theory for the five-defect model of alkali halide single crystals seem to exist. First there is the possiblity of some systematic experimental error. Second, another defect could provide an additional mechanism for ion or charge transport and thereby eliminate the apparent discrepancy between theory and experiment. Third the five-defect model may be adequate but the STIA and Lidiard theory may not be able to describe precisely the transport properties of $\mathrm{KCl}$ crystals. The correct explanation may be some combination of these three.

Regarding the first possibility, similar nonrandom deviations between conductivity data and the STIA have been reported for $\mathrm{KCl}$ by Beaumont and Jacobs, ${ }^{6}$ for $\mathrm{KBr}$ by Dawson and $\mathrm{Barr}^{23}$ and by Rolfe, ${ }^{24}$ and for the intrinsic region of $\mathrm{NaCl}$ by Allnatt and Pantelis. ${ }^{5}$ If these results are subjects to some systematic error this error is common to several laboratories. Since an error of this magnitude which is common to all groups and crystals is highly unlikely, we will henceforth ignore the possibility of systematic experimental errors.

Several other defects such as electrons, Frenkel defects, and trivacancies have been suggested as possible mechanisms for ion and/or electric charge transport. Assuming that the data presented in this paper are free from serious systematic errors, there are

\footnotetext{
${ }^{21}$ E. Koch and C. Wagner, Z. Physik Chem. (Leipzig) 38, 295 (1937).

${ }_{22}$ J. Teltow, Ann. Physik 5, 71 (1949).

${ }^{23} \mathrm{~L}$. W. Barr (private communication),

${ }^{24} \mathrm{~J}$. Rolfe (private communication).
}

three conditions that a single new mechanism would have to satisfy in order to explain the deviations of these data from the present theories. A new mechanism must (1) not change the qualitative features of the chlorine ion diffusion as a function of $T$ and $\mathrm{Sr}$ concentration, (2) make a contribution of at least $10 \%$ to the high-temperature $\left(T>700^{\circ} \mathrm{C}\right)$ conductivity of "pure" $\mathrm{KCl}$, and (3) produce more curvature in the extrinsic regions of the $\log (\sigma T)$ versus $10^{3} / T$ plots.

It is possible for electrons to be present in the conduction band of a $\mathrm{KCl}$ crystal, coming either from the metal electrode or from impurity traps in the crystal. In either case the concentrations of electrons would depend upon the individual sample, either through the electrode treatment or the impurity concentration. Yet the high-temperature intrinsic conductivity is precisely that part of the conductivity data which is reproducible from one laboratory to another and from one sample to another. ${ }^{4}$ Furthermore, the number of electrons required to remove the discrepancy between theory and experiment is extremely small, and would not be sufficient to alter the properties of the crystal in the extrinsic region as required by condition (3) above. It seems unlikely that electronic conduction is completely responsible for the discrepancy between theory and experiment.

The contribution of highly mobile interstitial potassium ions could explain the anomalous intrinsic conductivity and has been discussed by Allnatt and Pantelis. ${ }^{5}$ The presence of cation Frenkel defects could go unnoticed in the analysis of anion diffusion results, but such is not the case for precise cation diffusion data. For example, consider the results reported for "pure" $\mathrm{NaCl}$ by Allnatt and Pantelis. ${ }^{5}$ Their theoretical value using STIA for the conductivity of $\mathrm{NaCl}$ indicated that interstitial $\mathrm{Na}$ ions carried about $61 \%$ of the current at $794^{\circ} \mathrm{C}$. Such a large contribution of $\mathrm{Na}$ interstitials to the conductivity would make about $71 \%$ contribution to the cation diffusion if it occurred by the direct interstitial process or about $44 \%$ if by the interstitialcy mechanism. Unfortunately, precise cation diffusion data for $\mathrm{NaCl}$ near $794^{\circ} \mathrm{C}$ are not available. The presence of Frenkel defects, while possible, has not been demonstrated experimentally. The usefulness of a Frenkel defect mechanism to explain the deviations between theory and experiment as shown in Fig. 3 is questionable.

Trivacancies, of either the cation type $(+-+)$ or anion type $(-+-)$, could make a high-temperature contribution to the conductivity. It should be noted that the trivacancy concentration at constant temperature is directly proportional to the concentration of the isolated vacancy of a similar type. Hence, trivacancies could make a contribution to the conductivity in the extrinsic region [see condition (3) above]. A more 
complete discussion of a possible trivacancy contribution to conductivity is being published elsewhere. ${ }^{25}$

It seems probable that none of the above mechanisms can offer a complete solution to the problem created by discrepancy between the five-defect model and the experimental data. At least part of the cause of the discrepancy may lie in the simple assumptions that go into both the STIA and the Lidiard theory. For instance, it is difficult to justify a priori the assumption that the activation entropies and enthalpies are independent of temperature. It has been shown that the migration energy for vacancies in solid argon increases by $16 \%$ when the temperature is decreased by $20 \% .^{12}$ Such a large change in migration enthalpy would not be excepted for the alkali halide crystals, but thermal effects could be important. It is also assumed in the STIA and Lidiard theory that the vibrational contribution to the free energy is independent of the arrangements of the impurities and the vacancies. Preliminary results indicate that the differences between the vibrational states for complexes and isolated vacancies changes the temperature dependence for the concentration of complexes from the exponential form given by Eq. (7). ${ }^{26}$ Furthermore, the STIA completely neglects any longrange interaction between the defects. Lidiard's theoretical work showed the importance of including Coulomb interactions, but his results have found little use in subsequent analyses of experimental results. It has been recently shown that even the Lidiard theory, based on the Debye-Hückel approximations for elec-

${ }^{25}$ R. G. Fuller and M. H. Reilly, J. Phys. Chem. Solids (to be published).

${ }_{26}$ H. B. Rosenstock (private communication). trolytic solutions, underestimates the influence of defect interactions at high defect concentrations $(n \gtrsim 100 \mathrm{ppm})$, but more exact closed form expressions for the defect interactions that are readily applied to experimental results have not yet been obtained. If more careful treatments of the influence of defect interactions and lattice vibrations are included in the transport equations it may be possible to obtain a precise description of conductivity and diffusion on the basis of the five-defect model.

\section{SUMMARY}

In summary, there are nonrandom deviations between the experimental data and the transport equations derived from the five-defect model and the STIA and Lidiard theory. The usefulness of new mechanisms in explaining these deviations will have to be determined from results of additional experiments such as cation diffusion in pure $\mathrm{KCl}$ or ionic Hall effect. On the other hand, more complete theoretical treatment may remove the need for additional mechanisms to account for the discrepancy between present theoretical and experimental results.

\section{ACKNOWLEDGMENTS}

It is a pleasure to thank Dr. M. N. Kabler, Dr. H. B. Rosenstock, and Dr. C. C. Klick of their helpful comments; and Dr. R. J. Maurer, Materials Research Laboratory, University of Illinois, for sending us the $\mathrm{KCl}$ and $\mathrm{KCl}: \mathrm{SrCl}_{2}$ crystals on which the conductivity measurements were performed. We wish to thank Professor L. M. Slifkin for his suggestions concerning this manuscript. 